
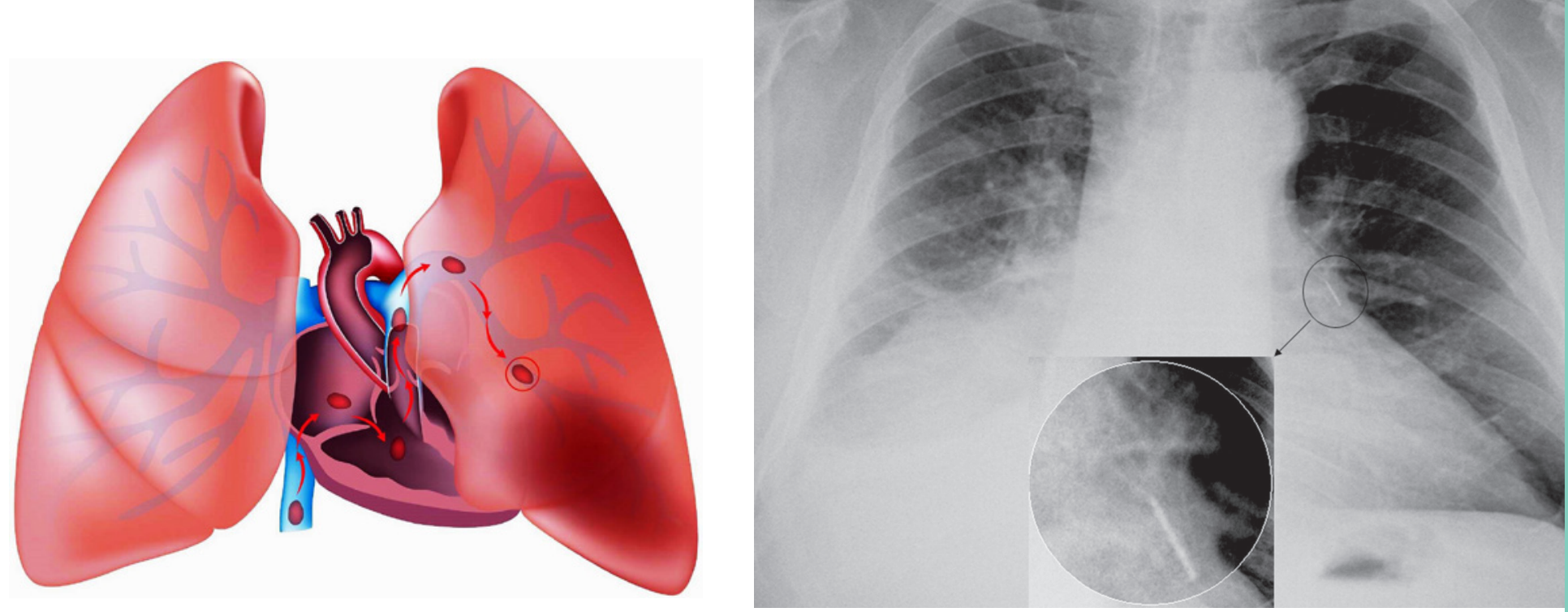

\title{
ESTUDIO DE CASO A UNA PERSONA CON ALTERACIÓN DE TROMBOEMBOLISMO PULMONAR Y NEUMONÍA
}

\author{
CASE STUDY OF A PERSON WITH ALTERATION OF LUNG AND PNEUMONIA THROMBOEMBOLISM
}

FRANCISCA LILIANA PALOMINO SALAZAR.*

\begin{abstract}
This case study was performed in critically ill adults with medical diagnosis of pulmonary thromboembolism submassive type I and community-acquired pneumonia, treated at the General Hospital of the State of Sonora. Evaluating functional patterns of Marjorie Gordon was used and supplemented with additional scales. Was used elaborated nursing diagnoses based on the NANDA Nursing Diagnoses Classification (NOC) and the Classification of Nursing Interventions (NIC) in care planning to perform. Clinical reasoning allows the application of relevant knowledge and assessment techniques, diagnostic and problem handling a patient. Therefore, from the above a care plan prioritizing the most affected needs and make individualized interventions was performed.
\end{abstract}

Keywords: Nursing situation, pulmonary embolism, pneumoni.
(*) Estudiante de Enfermería. Departamento de Enfermería. Universidad de Sonora. Email: fpalominosalazar@gmail.com

\section{RESUMEN:}

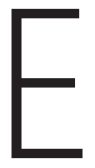
I presente estudio de caso se realizó a un adulto en estado crítico con diagnóstico médico de Tromboembolismo pulmonar submasivo tipo I y neumonía adquirida en la comunidad, atendido en un hospital público de Hermosillo, Sonora. Se utilizó la valoración de patrones funcionales de Marjorie Gordon, y se complementó con escalas adicionales. Se elaboraron los diagnósticos de Enfermería en base a la NANDA, así como las taxonomías para la Clasificación de Diagnósticos de Enfermería (NOC) y la Clasificación de las Intervenciones de Enfermería (NIC) para la planificación del cuidado. El razonamiento clínico permite la aplicación del conocimiento pertinente y las técnicas de evaluación, diagnósticos y manipulación del problema de un paciente. Por eso, a partir de lo anterior se realizó un plan de cuidados priorizando las necesidades así como intervenciones individualizadas.

Palabras clave: Situación de Enfermería, Tromboembolismo pulmonar, Neumonía. 



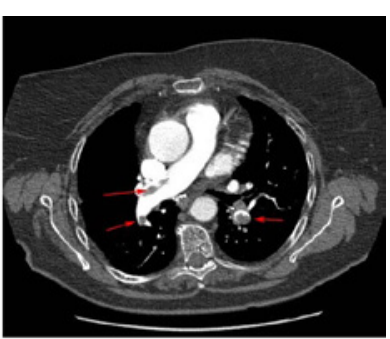

Este estudio de caso fue realizado en el área de Unidad de Cuidados Intensivos de una institución de segundo nivel. Se pretende aumentar las capacidades para razonar, resolver problemas y tomar decisiones en ámbitos de la vida del profesional de enfermería para lograr brindar cuidados de calidad.

\section{PRESENTACIÓN DEL CASO}

\section{Antecedentes}

Paciente masculino de 44 años de edad llega por su propio pie (autobús), inicia padecimiento el 8 de agosto de 2014 con signos de tos, disnea, cefalea y síncopes, taquicardia de 145 Ipm (los ha presentado desde hace 3 días), por lo que acude a un Centro de Salud siendo referido a un Hospital. Al realizarle la valoración inicial se encuentra con taquip-

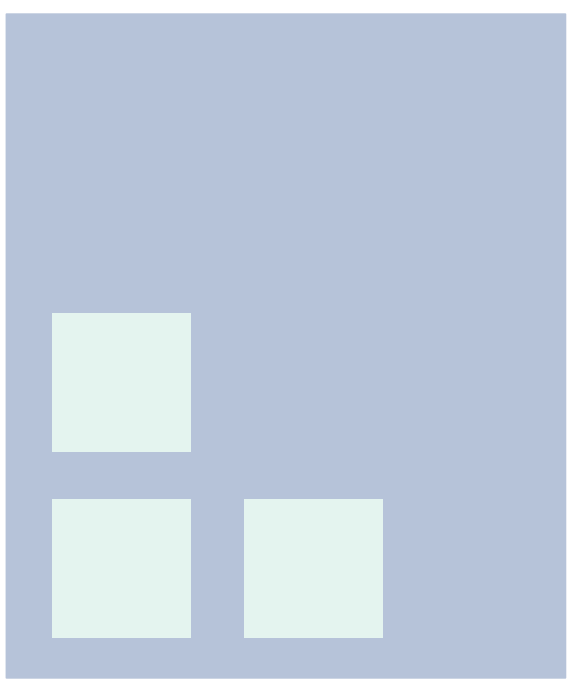

nea (32 rpm, superficiales), taquicárdico (137 lpm), pupilas isocóricas, con hiporreflexia, ansioso y diaforético; con respuesta a estímulos. Se pide valoración por el servicio de neumología y Rx, con el diagnóstico de Tromboembolismo pulmonar.

Padece diabetes mellitus tipo 2, hipertensión y cardiopatía congénita no identificada ambas sin tratamiento.

Posteriormente, se intuba y se le coloca catéter venoso central (CVC) en subclavia derecha, se instala sonda orogástrica (SOG) para alimentación y medicamentos. Y se ingresa al servicio de UCI.

\section{Heredofamiliares y patológicos}

Hermanos aparentemente sanos; padres finados. Madre padecía hipertensión arterial (HTA) y diabetes mellitus 2 (DM 2), padre con HTA y cardiopatía isquémica.

Al cuarto día de estar hospitalizado se diagnostica con Tromboembolismo pulmonar sub masivo tipo I en pulmón izquierdo y lóbulo inferior derecho y neumonía adquirida en la comunidad.

Se le indica el siguiente tratamiento:

- Solución Fisiológica 1000 ml, base a 41.6 gotas por minuto.

- Midazolam 15 ml/hora

- Fentanilo $10 \mathrm{ml} /$ hora

- $300 \mathrm{ml}$ de agua libre por las mañanas.

- Metroclopramida $10 \mathrm{mg}$.

- Enoxaparina 60 mg c/24 hr SC.

- Vancomicina 1gr IV cada 12 horas

- Amiodarona 200 mg SOG

- Cefepime c/12 hrs 1gr IV.

- Furosemida $10 \mathrm{mg}$ IV 12

- Metronidazol c/12 horas 500 mg IV 


\section{Valoración por patrones funcionales de Margory Gordon}

\section{Patrón de mantenimiento y percepción de la salud.}

Percibe su salud como mala, practicas higiénico dentales adecuadas e inadecuadas en la actividad física; con ingesta de bebidas alcohólicas y con toxicomanías negadas. Solicita atención médica debido a su enfermedad.

\section{Patrón nutricional metabólico.}

Presencia de sonda orogástrica con nutrición enteral (ensure) de $60 \mathrm{ml} / \mathrm{hora}$, pérdida de peso ( $5 \mathrm{~kg}$ en diez días), índice de masa corporal (IMC) de 27.78 $\mathrm{kg} / \mathrm{m} 2$, un perímetro abdominal de $110 \mathrm{~cm}$., peristalsis aumentada (+ $\left.40 \mathrm{x}^{\prime}\right)$. Se observa lesión en región occipital (úlcera por presión grado III, de 5 cms de diámetro), presencia de caries en la mayoría de las piezas dentarias (28 piezas dentarias), presencia de halitosis, edema en labios y sialorrea. Piel reseca, deshidratada con poca turgencia, eutérmico, y edema en miembros inferiores (fóvea de +++ ).

Los laboratorios reportan leucocitosis, eritropenia, hipocloremia, anemia ( $\mathrm{Hb}$ 8.9) y hematocrito bajo (28.9).

\section{Eliminación.}

Evacuaciones diarreicas color verdoso con moco (7 escala de Bristol); Orina turbia (ámbar) con sedimentos, presencia de catéter vesical, con diuresis horaria de 0.92 $\mathrm{ml} / \mathrm{kg} / \mathrm{hr}$ sin datos de infección en meato urinario. Presenta diaforesis. Pérdidas insensibles de 1080, con balance +303 .

\section{Patrón de actividad y ejercicio.}

Con ventilación mecánica (VM) en modalidad asisto controlado con 16 rpm, cánula orotraqueal, con parámetros de $\mathrm{PaO} 266 \mathrm{mmHg}$, $\mathrm{PaCO} 251 \mathrm{mmHg}$, PEEP $13 \mathrm{cmH} 2 \mathrm{O}$, Vte 0.63, Ti: 1.24 seg, FiO2 70\%, Vt 10.2, relación IE 1:2.; campos pulmonares con ruidos agregados (estertores en hemitórax izquierdo), hipoaereación en región derecha a nivel de base; gases arteriales reportan, hipoxemia, y una alcalosis metabólica compensada. Presentando taquicardia supra ventricular, con presión arterial media de $90 \mathrm{mmHg}$, presión venosa central de $18 \mathrm{cmH} 2 \mathrm{O}$. Su capacidad de movilización comprometida por efectos de la sedación, presentando un alto riesgo de ulceras por presión según la escala análoga de Braden Bergstrom y un alto riesgo de caídas según la escala de morse.

Cuenta con ECG donde se muestra el patrón McGuinn - White donde se encuentra S1, Q3, T3. Complejos QRS anchos (> 12 segundos). P pulmonar + BRD (ICC derecha) por hipertrofia de VD.

En Rx de tórax se observa una moderada disminución del volumen pulmonar izquierdo, con ascenso de hemidiafragma y colapso del lóbulo inferior izquierdo, compatible con focos de atelectasia. También se observan sombras similares en lóbulo inferior derecho.

\section{Sueño - descanso.}

No presenta estímulos (Ramsey 6).

\section{Cognitivo perceptivo.}

Presenta hiporreflexia en ambos ojos ante la luz. Pupilas isocóricas.

\section{Patrón de sexualidad y repro- ducción.}

Región perianal hiperémica, no circundado, meato limpio; ausencia de reflejo cremasteriano. Integridad en mamas, simétricas tipo I. Cadena ganglionar.

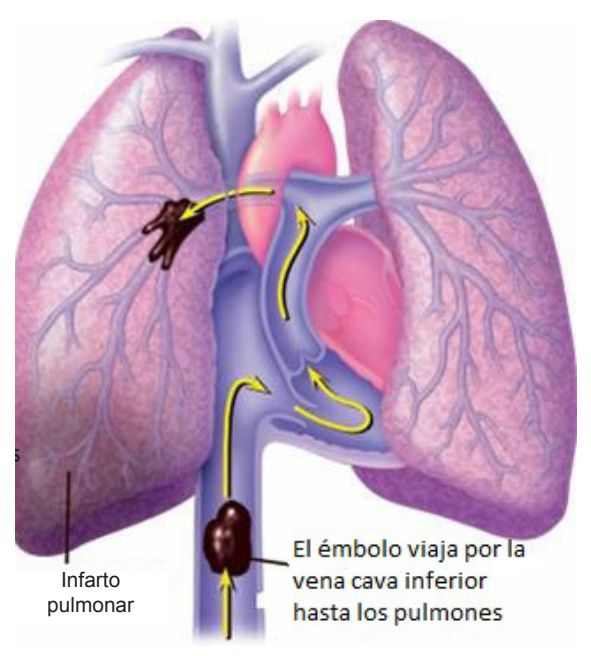

No tiene hijos 


\begin{tabular}{|c|c|}
\hline Patrón funcional de salud & Etiqueta Dx \\
\hline Actividad - ejercicio & $\begin{array}{l}\text { DOMINIO 4: Actividad/Reposo } \\
\text { CLASE 4: Respuesta cardiovascular / Pulmonar } \\
\text { (00092) Disminución del gasto cardíaco R/C alteración de la precarga y alteración de la frecuencia cardíaca M/P } \\
\text { aumento de la PVC y edema. }\end{array}$ \\
\hline Actividad - ejercicio & $\begin{array}{l}\text { DOMINIO 11: Seguridad / protección } \\
\text { CLASE 2: Lesión física. } \\
\text { (00031) Limpieza ineficaz de las vías aéreas R/C vía aérea artificial y mucosidad excesiva M/P sonidos respiratorios } \\
\text { adventicios (estertores diseminados) cambios en la frecuencia respiratoria. }\end{array}$ \\
\hline Eliminación & $\begin{array}{l}\text { DOMINIO 2: Nutrición } \\
\text { CLASE 5: Hidratación. } \\
\text { (00026) Exceso del volumen de líquidos R/C compromiso de los mecanismos reguladores M/P edema, aumento de la } \\
\text { PVC y sonidos respiratorios ocasionales. }\end{array}$ \\
\hline Eliminación & $\begin{array}{l}\text { DOMINIO 3: Eliminación e intercambio } \\
\text { CLASE 2: Función gastrointestinal. } \\
\text { (00196) Motilidad gastrointestinal disfuncional R/C medicamentos (sedantes) y nutrición enteral (malnutrición) M/P } \\
\text { diarrea y cambio en ruidos intestinales (hiperactivos). }\end{array}$ \\
\hline Actividad - Ejercicio & $\begin{array}{l}\text { DOMINIO 3: Eliminación e intercambio } \\
\text { CLASE 4: Función respiratoria. } \\
\text { (00030) Deterioro del intercambio de gases R/C ventilación-perfusión M/P color anormal de la piel (palidez) y } \\
\text { gasometría arterial anormal. }\end{array}$ \\
\hline Nutricional Metabólico & $\begin{array}{l}\text { DOMINIO 11: Seguridad y protección. } \\
\text { CLASE 2: Lesión física. } \\
\text { (00045) Deterioro de la mucosa oral R/ factores mecánicos (intubación endotraqueal y sonda orogástrica) M/P } \\
\text { lesiones orales, edema (macroglosia) y halitosis. }\end{array}$ \\
\hline Nutricional Metabólico & $\begin{array}{l}\text { DOMINIO 11: Seguridad / protección } \\
\text { CLASE 2: Lesión física. } \\
\text { (00044) Deterioro de la integridad tisular R/C factores nutricionales (déficit) y deterioro en la circulación M/P } \\
\text { destrucción tisular (UPP en occipital y región glútea y sacra). }\end{array}$ \\
\hline
\end{tabular}

DOMINIO 11: Seguridad / protección

CLASE 2: Lesión física.

DIAGNÓSTICO ENFERMERO (NANDA): (00031) Limpieza ineficaz de las vías aéreas R/C vía aérea artificial (TET) y mucosidad excesiva (sialorrea) M/P sonidos respiratorios adventicios (estertores diseminados).

\begin{tabular}{|l|c|}
\hline \multicolumn{1}{|c|}{ INDICADORES } & HORARIOS \\
\hline - 041007 Ruidos respiratorios patológicos. & $9: 30 \mathrm{hrs}$ \\
- 041020 Acumulación de esputos. & $10: 00 \mathrm{hrs}$ \\
- 041012 Capacidad de eliminar secreciones. & $10: 00 \mathrm{hrs}$ \\
\hline
\end{tabular}

\begin{tabular}{|c|c|}
\hline INTERVENCIONES (NIC) & ACTIVIDADES \\
\hline $\begin{array}{l}3160 \text { - Aspiración de las vías aéreas. } \\
\text { - Determinar la necesidad de la aspiración oral y/o traqueal. } \\
\text { - Hiperoxigenar al } 100 \% \text { cuando se realice aspiración. } \\
\text { - Anotar el tipo y cantidad de secreciones obtenidas. } \\
\mathbf{3 1 4 0} \text { - Manejo de las vías aéreas. } \\
\text { - Colocar al paciente en la posición que permita que el potencial de } \\
\text { ventilación sea al máximo posible. } \\
\text { - Realizar fisioterapia pulmonar. } \\
\text { - Auscultar sonidos respiratorios, observando las áreas de disminución } \\
\text { o ausencia de ventilación y la presencia de sonidos adventicios. } \\
\text { - Vigilar el estado respiratorio y de oxigenación. } \\
\mathbf{3 1 8 0} \text { - Manejo de las vías aéreas artificiales. } \\
\text { - Asegurar que el globo del TET este inflado y no tenga fugas. }\end{array}$ & $\begin{array}{l}\text { - Auscultación de campos pulmonares para ver la existencia de } \\
\text { secreciones. } \\
\text { - Brindar altas concentraciones de oxígeno cuando se lleve a cabo } \\
\text { la aspiración. } \\
\text { - Registro de actividades realizadas. } \\
\text { - Se coloca al paciente es semifowler. } \\
\text { - Se da posición al paciente para poder movilizar secreciones y } \\
\text { lograr su excreción. } \\
\text { - Monitorización de estado respiratorio. } \\
\text { - Verificar inflado de globo de TET para evitar fugas. }\end{array}$ \\
\hline \multicolumn{2}{|c|}{ EVALUACIÓN } \\
\hline
\end{tabular}




\section{DOMINIO 2: Nutrición}

\section{CLASE 5: Hidratación}

DIAGNÓSTICO ENFERMERO (NANDA): (00026) Exceso de volumen de líquidos R/C compromiso de los mecanismos reguladores (Hemodinamicamente inestable) $\mathrm{M} / \mathrm{P}$ edema, aumento de la $\mathrm{PVC}\left(18 \mathrm{cmH}_{2} \mathrm{O}\right)$ y sonidos respiratorios ocasionales (estertores), disminución de hematocrito (28.9) y disminución de hemoglobina ( $8.9 \mathrm{~g} / \mathrm{dL})$.

\begin{tabular}{|l|c|}
\hline \multicolumn{1}{|c|}{ INDICADORES } & HORARIOS \\
\hline - 060103 Presión venosa central. & Horaria. \\
- 060107 Entradas y salidas diarias equilibradas. & Uno por turno. \\
- 060116 Hidratación cutánea. & $9: 00$ horas. \\
- 060112 Edema periférico. & $8: 00$ horas. \\
\hline
\end{tabular}

\begin{tabular}{|c|c|}
\hline INTERVENCIONES (NIC)/ACTIVIDADES & ACTIVIDADES \\
\hline $\begin{array}{l}\text { - Tener un control estricto de ingreso y egreso de líquidos (balance hídrico positivo } \\
+303 \mathrm{ml} \text { ). } \\
\text { - Hacer balance de ingresos y egresos. } \\
\text { - Pérdidas insensibles. } \\
\text { - Administración de medicamentos para eliminar el exceso de líquidos (Furosemida } \\
10 \mathrm{mg} \text { ). } \\
\text { - Aplicarle crema humectante en la piel para evitar resequedad (se aplica vaselina } \\
\text { para la piel). } \\
\text { - Observar la coloración y cantidad de la orina (mantiene anuria). } \\
\text { - Monitorización cardíaca y signos vitales horarios. } \\
\text { - Consultar con el médico tratante si los signos no mejoran o empeoran (se comunica } \\
\text { a médico tratante signos vitales, pvc de paciente no hace cambios). } \\
\text { - Monitorizar el estado de hemodinamia (PVC, PAM). } \\
\text { - Valorar la piel, la cara y partes declives por si presenta edema. } \\
\text { - Evaluar el grado de edema. } \\
\text { - Auscultar ruidos pulmonares y cardíacos (se ausculta pulmón izquierdo } \\
\text { hipoventilado). }\end{array}$ & $\begin{array}{l}\text { - Llevar un control estricto de } \\
\text { líquidos. } \\
\text { - Evitar que al paciente le dé } \\
\text { hipertermia. } \\
\text { - Administración de medicamentos } \\
\text { diuréticos. } \\
\text { - Aplicar crema para la piel. } \\
\text { - Observar características de la orina } \\
\text { y descartar complicaciones. } \\
\text { - Toma y registro de signos vitales } \\
\text { horaria } \\
\text { - Toma de PVC horaria } \\
\text { - Realizar prueba (hundimiento de } \\
\text { dedos) para valorar fóvea. } \\
\text { - Auscultación de campos } \\
\text { pulmonares y focos cardíacos. }\end{array}$ \\
\hline
\end{tabular}

\section{EVALUACION}

Se tuvo control de ingreso y egreso de líquidos. Se le administró diurético de asa (furosemide) 10 mg para la acumulación de líquido sin mucha mejora tras la administración. Se observa orina con presencia de sedimentos color ámbar lo cual se reporta al médico tratante, no hace cambios; sus signos vitales están estables con alteraciones ocasionales en la movilización (aumento de la FC). Su PVC horaria se muestra elevada entre 17-18 $\mathrm{cmH}_{2} \mathrm{O}$ indicando ICC pudiéndosele atribuir al exceso de líquidos por ello la importancia de su cuantificación. Se le aplica humectante para la piel. Paciente lleva un cuidado adecuado.

\section{CONCLUSIÓN}

El estudio de caso permite al profesional de enfermería la comprensión de las características particulares de las personas, además facilita la obtención de información desde diferentes perspectivas, considerando su ciclo de vida y realidad social Este caso, ha sido de ayuda para dar a conocer un estudio de caso abordado a través del proceso de enfermería (PE) mediante el pensamiento crítico en la toma de decisiones independientes de enfermería así como en el trabajo multidisciplinario. Este trabajo se basa en el marco de valoración por patrones funcionales de Margory Gordon, lo cual ayuda a identificar fortalezas y debilidades que el paciente presenta para así brindar un cuidado oportuno y de calidad. Así mismo trabajo que nos permite de una manera acertada realizar diagnósticos de enfermería y enlistar las actividades/intervenciones de actuación que forman parte de una recuperación del paciente o en su lugar evitan que surjan complicaciones de cualquier índole según el padecimiento.

En este caso es importante considerar la posibilidad de recurrencias tromboembólicas que agraven el cuadro y aceleren el colapso hemodinámico de un paciente previamente estable.

Las posibilidades de éxito terapéutico de la neumonía adquirida en la comunidad están en la precocidad con la que se inicia el tratamiento y de la correcta identificación del cuadro clínico para poder definir un tratamiento ambulatorio o la necesidad de soporte hospitalario. $(7,12)$

Los costos que genera el cuidado de estas enfermedades han ido en aumento progresivamente, concentrándose en los pacientes que están hospitalizados en UCI, por lo que es de vital importancia la función de las enfermeras al identificar precozmente los problemas de salud de sus usuarios.

\section{REFERENCIAS BIBLIOGRÁFICAS.}

1. Gómez ER. Fundamentos de enfermería: Ciencia, metodología y tecnología: Editorial El Manual Moderno; 2015.

2. Orkaizagirre Gómara A, Amezcua $M$, Huércanos Esparza I, Arroyo Rodríguez A. El Estudio de casos, un instrumento de aprendizaje en la Relación de Cuidado. Index de Enfermería. 2014;23(4):244-9.

3. Coto Vargas N, Molina Chaves R, Rivera Soto R. Intervencion de enfermeria obstetrica: un estudio de caso. 2013.

4. Ferri FF. Ferri consultor clínico, 2006-2007: claves diagnósticas y tratamiento: Elsevier; 2006.

5. Santos Martínez LE, Uriona Villarroel JE, Exaire Rodríguez JE, Mendoza D, Martínez Guerra ML, Pulido T, et al. Tromboembolia pulmonar masiva, trombo en tránsito y disfunción ventricular derecha. Archivos de cardiología de México. 2007;77(1):4453.

6. Morales-Blanhir JE, Salas-Pacheco JL, Rosas-Romero MdJ, Valle-Murillo MÁ. Diagnóstico de tromboembolia pulmonar. Archivos de cardiología de México. 2011;81(2):126-36.

7. Jerjes-Sánchez C, Ramírez-Rivera A. Tromboembolia pulmonar. Recomendaciones terapéuticas de las guías clínicas de la Sociedad Mexicana de Cardiología. Arch Cardiol Mex. 2007;77(S4):202-6.

8. Aizman A, Mercado M, Andresen M. Tromboembolismo pulmonar: estratificación de riesgo y dilemas terapéuticos. Revista médica de Chile. 2012;140(11):1482-9.

9. Torbicki A. Enfermedad tromboembólica pulmonar. Manejo clínico de la enfermedad aguda y crónica. Revista española de cardiología. 2010;63(7):832-49.

10. Báez Saldaña R, Gómez Zamora C, López Elisondo C, Molina Corona H, Santillán Martínez A, Sánchez Hernández J. Neumonía adquirida en la comunidad. Revisión y actualización con una perspectiva orientadora a la calidad de la atención médica. Neumol Cir Torax. 2013;72(Supl 1):6-43.

11. Valdivia C. Epidemiología de la neumonía del adulto adquirida en la comunidad. Revista chilena de infectología. 2005;22:s11-s7.

12. Guía de práctica clínica para prevención diagnóstico y tratamiento de la endocarditis infecciosa de la Sociedad GdT (nueva versión 2009). 2009. 